\title{
A cross-sectional study on uric acid levels among Chinese adolescents
}

\author{
Jie Lu ${ }^{1,2,3}$ - Wenyan Sun ${ }^{1,2,3} \cdot$ Lingling Cui ${ }^{1,2,3} \cdot$ Xinde $\mathrm{Li}^{1,2,3} \cdot$ Yuwei He ${ }^{1,2,3} \cdot$ Zhen Liu ${ }^{1,2,3} \cdot$ Hailong $\mathrm{Li}^{1,2,3}$.

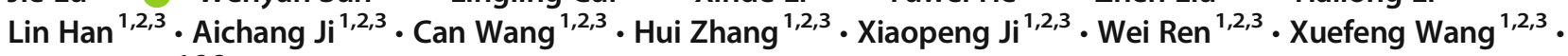 \\ Changgui $\mathrm{Li}^{1,2,3}$
}

Received: 30 May 2019 /Revised: 6 August 2019 / Accepted: 6 September 2019 / Published online: 6 December 2019

(C) The Author(s) 2019

\begin{abstract}
Background The prevalence of hyperuricemia is increasing in adults, while the prevalence among adolescents is seldom reported.

Methods A cross-sectional survey by multistage, stratified sampling method was carried out in Shandong Province during 20172018. A total of 9371 adolescents aged from 13 to 19 years were randomly sampled and analyzed in this survey.

Results The overall mean serum uric acid (sUA) concentration was $6.08 \pm 1.57 \mathrm{mg} / \mathrm{dL}$ and overall hyperuricemia prevalence was $25.4 \%$ and $60.5 \%$ (when hyperuricemia was defined as sUA $\geq 7 \mathrm{mg} / \mathrm{dL}$ or $\geq 5.5 \mathrm{mg} / \mathrm{dL}$ ). Prevalence were $42.3 \%$ (male) and $8.0 \%$ (female) when limit was $7 \mathrm{mg} / \mathrm{dL}$ and prevalence were $82.1 \%$ (male) and $38.4 \%$ (female) when limit was $5.5 \mathrm{mg} / \mathrm{dL}$. Male gender, increased body mass index, increased waist circumstance, increased triglycerides, increased fasting blood glucose, increased systolic blood pressure, decreased estimated glomerular filtration rate, and positive family gout history were associated with the enhanced risk of hyperuricemia according to univariate and/or multivariate logistic regression analysis. Food intake frequency of carbonate beverage, mutton, and other kinds varied between hyperuricemia adolescents and normal sUA ones. Conclusions The studied adolescent population showed sUA level and hyperuricemia prevalence which are even higher than those of adults in China. The epidemic of youth hyperuricemia may pose a future threat of gout attacks and other hyperuricemiarelated diseases, which alarms the public, health professionals and health policy makers to prepare the future health challenges.
\end{abstract}

Keywords Prevalence $\cdot$ Hyperuricemia $\cdot$ Adolescents

\section{Introduction}

Hyperuricemia is a major pathogenic factor for urate deposition diseases (gouty arthritis, renal disorder, etc.). In addition to gout, hyperuricemia is the risk factor of a series of diseases including ischemic heart disease, diabetes mellitus, lipid abnormalities, hypertension, stroke, and preeclampsia [1]. Gout and hyperuricemia affect predominantly males since serum

Jie Lu, Wenyan Sun and Lingling Cui contributed equally to this work.

Changgui Li

lichanggui@medmail.com.cn

1 Shandong Provincial Key Laboratory of Metabolic Diseases and Qingdao Key Laboratory of Gout, the Affiliated Hospital of Qingdao University, Qingdao, China

2 Department of Endocrinology and Metabolism, the Affiliated Hospital of Qingdao University, Qingdao, China

3 Institute of Metabolic Diseases, Qingdao University, Qingdao 266003, China uric acid (sUA) are considerably lower in women. Since gout is considered as an adult disease and hyperuricemia is asymptomatic in most people, pediatric hyperuricemia is often overlooked. Recent meta-analysis showed the prevalence of hyperuricemia and gout in mainland China reached 13.3\% and $1.1 \%$ respectively [2]. However, it still lacks large sample-based reports on the prevalence of hyperuricemia among Chinese adolescents. The current study aimed to investigate the prevalence of hyperuricemia among adolescents (13-19 years old) from Shandong Province, one of the most populous provinces of China.

\section{Methods}

In this study, we conducted a population-based cross-sectional study of enrolled students from the representative middle schools and universities of Shandong Province during 2017-2018. We used a multistage, stratified sampling method to obtain a representative sample of people 
aged 13-19 years in the general population. We randomly selected two urban and two rural districts (defined according to the National Bureau of Statistics in China) in Shandong Province. In the second stage, clusters of two schools were randomly selected from each district using probability proportional to size sampling. To minimize sampling bias due to age-dependent physiological change of urate level during puberty and life-style-related factors associated with the typical urban-rural dual system of China, we took stratified sampling strategy by matching the numbers of participants according to age and residence (rural or urban). In the third stage, individuals were randomly chosen (by simple randomization using SPSS software) from each school in each stratum. Only official registered students were enrolled and we excluded participants without blood samples and pregnant girls. Targeting participants were informed not to alter their diet or physical activity for at least 3 days before the examination. The study protocol was approved by the ethics review committee of the Affiliated Hospital of Qingdao University (\#QYFY WZLL 25565). Participants 18 years or older provided written consent. Both written assent and parental permission were obtained in writing for youth younger than 18 years.

Body weight and height were measured according to a standard protocol. Body mass index (BMI) was calculated as weight in kilograms divided by height in meters squared. Blood samples were collected after overnight fasting. Serum levels of uric acid, creatinine, urea nitrogen, glucose, total cholesterol (TC), triglycerides (TG), and high-density lipoprotein-cholesterol (HDL-C) were measured enzymatically using an automatic analyzer (Toshiba, Tokyo, Japan) at the Gout Laboratory of the Affiliated Hospital of Qingdao University.

Since no previous standard of hyperuricemia for adolescents has been established, we defined hyperuricemia based on both putative adult hyperuricemia threshold $(7.0 \mathrm{mg} / \mathrm{dL})$ and hyperuricemia threshold among adolescents $(5.5 \mathrm{mg} /$ dL) reported previously [3]. To define the metabolic syndrome (MetS) in adolescents, we used a previously proposed modification of the definition by the International Diabetes Federation (IDF) [4]. Accordingly, obesity was defined as waist circumference $\geq 90$ th percentile among peer group, hyperlipidemia as triglycerides $\geq 1.7 \mathrm{mg} / \mathrm{dL}$; hyperglycemia as fasting blood glucose $\geq 5.6 \mathrm{mmol} / \mathrm{L}$. We defined hypertension as $\geq 130 \mathrm{mmHg}$ systolic or $\geq$ $80 \mathrm{mmHg}$ diastolic by guidelines of 2017 American Academy of Pediatrics (AAP) [5] for participants aged from 13 to 18 years and 2017 American College of Cardiology/ American Heart Association (2017 ACC/AHA) [6] for 19year-old adolescents. Estimated glomerular filtration rate (eGFR; $\mathrm{ml} / \mathrm{min}$ per $1.73 \mathrm{~m}^{2}$ ) was calculated via the serum creatinine (Scr)-based formula of the full age spectrum (FAS) [7, 8]: 107.3/[Scr/Q] where
$Q=0.23+0.034 \times$ age $-0.0018 \times$ age $^{2}+0.00017 \times$ age $^{3}-$ $0.0000051 \times$ age $^{4}$, for girls $<18$ years;

$Q=0.21+0.057 \times$ age $-0.0075 \times$ age $^{2}+0.00064 \times$ age $^{3}-$ $0.000016 \times$ age $^{4}$, for boys $<18$ years; $Q=0.70$ for female patients when age $\geq 18$ years; $Q=0.90$ for male patients when age $\geq 18$ years

During recruitment, participants completed a validated food frequency questionnaire in which participants were asked to answer the question "How often, on average, in the past weeks did you eat [this food]?" These categorical answers were converted to average servings per week for analysis. Food intake frequencies were converted into conversion factors. Specifically, $1-3$ servings/month was converted to 0.47 , 1 serving/week converted to 1 , and 2-4 servings/week converted to 3 .

Data were expressed as median and interquartile range (IQR). Prevalence and 95\% confidence interval (CI) of hyperuricemia were estimated overall and stratified by sex and age. Chi-square test was applied in group comparison. Binary logistic regression was used to assess the associations between hyperuricemia and the related risk factors in univariable and multivariable analyses. Student's $t$ test was applied in the comparison of food frequency. We did not impute missing data. Analyses were conducted using R statistics (version 3.4.16) and SPSS (version 19.0). $p<0.05$ for a two-tailed test was considered statistically significant.

\section{Results}

The survey was completed by 9371 respondents out of the targeted 11,602 Chinese adolescents, giving the survey a good response rate of $80.8 \%$. The final sample included $50.6 \%$ boys and $49.3 \%$ girls. The students' ages ranged from 13 to 19 years old, with the median age of 16 years old. The general demographic and clinical characteristics were compared between hyperuricemia and normal sUA participants (Table 1). The overall median sUA level was 5.92 (IQR, 4.96-7.03) mg/ dL (6.72 (IQR, 5.83-7.71) mg/dL for boys and 5.18 (4.47$5.97) \mathrm{mg} / \mathrm{dL}$ for girls). When hyperuricemia was defined as $\mathrm{sUA} \geq 7.0 \mathrm{mg} / \mathrm{dL}$, the overall hyperuricemia prevalence was $25.4 \%$ and prevalence in boys and girls were $42.3 \%$ and $8.0 \%$ respectively. When defined as sUA $\geq 5.5 \mathrm{mg} / \mathrm{dL}$, the overall hyperuricemia prevalence was strikingly $60.5 \%$ and prevalence in boys and girls were $82.1 \%$ and $38.4 \%$ respectively (Fig. 1a).

Logistic regression analysis revealed male gender, obesity, and metabolic syndrome-related variables such as BMI, WC, TG, FBG, SBP were associated with the enhanced risk of hyperuricemia. Noticeably, positive family gout history was also found involved in the association of hyperuricemia risk by multivariate logistic regression analysis (Fig. 1b, c). Carbonate beverage, mutton, and sugar-sweetened beverage 
Table 1 Demographic and general clinical characteristics of the participants according to sUA levels (hyperuricemia vs. normal sUA)

\begin{tabular}{|c|c|c|c|c|}
\hline Variables & Total & HUA & Normal sUA & $P$ value \\
\hline $\mathrm{N}$ & 9371 & 2282 & 7089 & \\
\hline Age, years & $16(15-18)$ & $16(15-18)$ & $16(15-18)$ & $>0.05$ \\
\hline $\mathrm{sUA}, \mathrm{mg} / \mathrm{dL}$ & $5.92(4.96-7.03)$ & $7.92(7.41-8.71)$ & $5.43(4.69-6.17)$ & $<0.001$ \\
\hline 13 & $5.66(4.74-6.77)$ & $7.88(7.38-8.45)$ & $5.28(4.55-6.03)$ & $<0.001$ \\
\hline 14 & $5.56(4.74-6.69)$ & $7.98(7.42-8.65)$ & $5.24(4.54-5.97)$ & $<0.001$ \\
\hline 15 & $6.10(5.14-7.18)$ & $7.82(7.41-8.64)$ & $5.58(4.82-6.25)$ & $<0.001$ \\
\hline 16 & $6.17(5.28-7.36)$ & $8.00(7.51-8.89)$ & $5.66(4.98-6.25)$ & $<0.001$ \\
\hline 17 & $5.83(4.84-6.92)$ & $7.83(7.41-8.57)$ & $5.38(4.59-6.20)$ & $<0.001$ \\
\hline 18 & $5.88(4.96-7.07)$ & $8.01(7.36-8.69)$ & $5.43(4.66-6.22)$ & $<0.001$ \\
\hline 19 & $5.88(4.96-6.97)$ & $7.93(7.37-8.80)$ & $5.41(4.67-6.12)$ & $<0.001$ \\
\hline BMI, $\mathrm{kg} / \mathrm{m}^{2}$ & $20.4(18.5-23.0)$ & $22.2(19.6-22.1)$ & $20.0(18.3-26.0)$ & $<0.001$ \\
\hline \multicolumn{5}{|l|}{ Urbanization } \\
\hline Urban, $n(\%)$ & $1866(46.3)$ & $431(47.0)$ & $1435(46.1)$ & $>0.05$ \\
\hline Waist circumference, $\mathrm{cm}$ & $72.0(67.0-79.0)$ & $78.0(71.0-88.0)$ & $70.0(66.0-77.0)$ & $<0.001$ \\
\hline Obesity, $n(\%)$ & $449(4.8)$ & $278(12.2)$ & $171(2.4)$ & $<0.001$ \\
\hline Hyperlipidemia, $n(\%)$ & $317(3.4)$ & $117(5.1)$ & $200(2.8)$ & $<0.001$ \\
\hline Hyperglycemia, $n(\%)$ & $47(0.8)$ & $17(1.2)$ & $30(0.7)$ & $>0.05$ \\
\hline Hypertension, $n(\%)$ & 2037 (21.7) & $733(32.1)$ & $1304(18.4)$ & $<0.001$ \\
\hline Metabolic syndrome, $n(\%)$ & $20(0.5)$ & $17(0.7)$ & $3(0.2)$ & $<0.001$ \\
\hline $\mathrm{eGFR}, \mathrm{ml} / \mathrm{min} / 1.73 \mathrm{~m}^{2}$ & $102.2(89.5-115.0)$ & $95.3(85.4-106.6)$ & $104.7(91.9-117.2)$ & $<0.001$ \\
\hline$\leq 75 \mathrm{ml} / \mathrm{min} / 1.73 \mathrm{~m}^{2}, n(\%)$ & $490(5.2)$ & $142(6.2)$ & $348(4.9)$ & 0.014 \\
\hline Family history of gout, $n(\%)$ & $212(3.1)$ & $56(3.5)$ & $156(3.0)$ & $>0.05$ \\
\hline *Menarche age, years & $12(12-13)$ & $12(11-13)$ & $13(12-13)$ & $<0.001$ \\
\hline *Irregular menstruation, $n(\%)$ & $1096(41.2)$ & $113(45.2)$ & $983(40.8)$ & $<0.001$ \\
\hline
\end{tabular}

Data are presented as median (IQR, interquartile range). sUA, serum uric acid; HUA, hyperuricemia (sUA $\geq 7.0$ $\mathrm{mg} / \mathrm{dL}$ ); BMI, body mass index; eGFR, estimated glomerular filtration rate. Obesity was defined as waist circumference $\geq 90$ th percentile among peer group; hyperlipidemia was defined as triglycerides $\geq 1.7 \mathrm{mg} / \mathrm{dL}$; hyperglycemia defined as fasting blood glucose $\geq 5.6 \mathrm{mmol} / \mathrm{L}$; hypertension $\geq 130 \mathrm{mmHg}$ systolic or $\geq$ $80 \mathrm{mmHg}$ diastolic; metabolic syndrome was defined as a proposed modification of the definition by the International Diabetes Federation. eGFR $\left(\mathrm{ml} / \mathrm{min}\right.$ per $1.73 \mathrm{~m}^{2}$ ) was calculated via the age-based formula of the Full Age Spectrum. Irregular menstruation delegates girls with irregular periods *Data were calculated based on females were the top three foods with a significant difference in consumption frequency between adolescents with hyperuricemia and with normal sUA (Fig. 1d). Food like steamed bun, pork, beef, etc. displayed significant differences in intake frequency as well $(p<0.05)$.

\section{Discussion}

The current study sent an alarm of a pressing epidemic of hyperuricemia among adolescents in China. Considering reported prevalence (adults or overall population) of asymptomatic hyperuricemia is around $13.3 \%$ in China and between 4 and $25 \%$ in major countries of the world [3] and puberty uric acid (normally 3-4 mg/dL) levels are considerably lower than the adults [9], the results unequivocally revealed a hyperuricemia epidemic is happening in Shandong Province or beyond. We identified male gender, increased BMI, increased waist circumstance, increased TG, increased FBG, increased SBP, decreased eGFR, and positive family gout history were associated with the enhanced risk of hyperuricemia, which are similar with risk factors among adults [3].

In the 1999-2006 National Health and Nutrition Examination Survey, 6036 adolescents 12 to 17 years of age were included to analyze the relationship between uric acid and blood pressure [10]. In the adjusted analysis, the odds ratio of elevated blood pressure, for each $0.1 \mathrm{mg} / \mathrm{dL}$ increase in uric acid level was 1.38 (95\% CI, 1.16 to 1.65). Compared with $<5.5 \mathrm{mg} / \mathrm{dL}$, participants with a uric acid level $\geq 5.5 \mathrm{mg} /$ $\mathrm{dL}$ had a 2.03 fold (95\% CI, 1.38 to 3.00 ) elevated blood pressure. Similarly, the proportion of hypertension in the hyperuricemia group was much higher than that in the normal sUA group and the elevated SBP was related to enhanced risk of hyperuricemia in our study. As we defined adolescent hypertension as $\geq 130 \mathrm{mmHg}$ systolic or $\geq 80 \mathrm{mmHg}$ diastolic by the 2017 AAP and 2017 ACC/AHA guidelines in this 
a

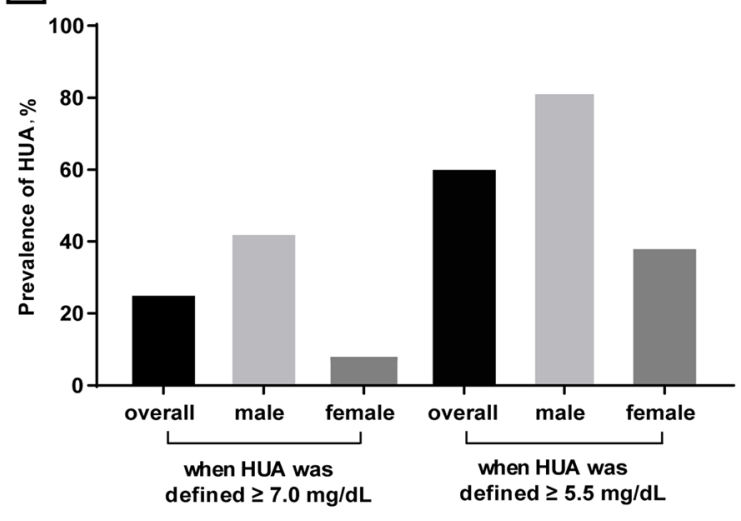

b
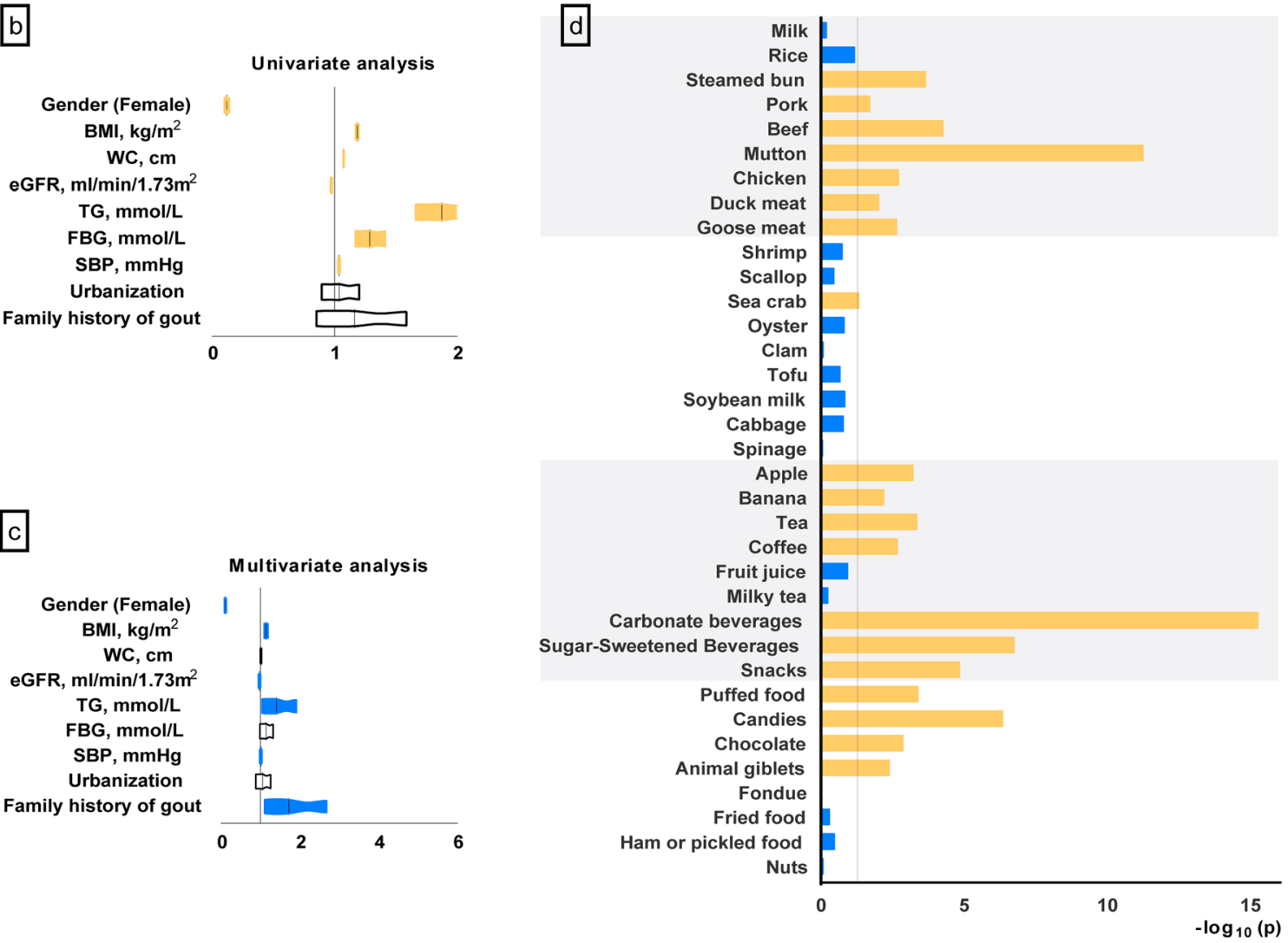

Fig. 1 Summary of the hyperuricemia prevalence among Chinese adolescents and associated factors with hyperuricemia. sUA, serum uric acid; HUA, hyperuricemia; BMI, body mass index; WC, waist circumference; TC, total cholesterol; TG, triglycerides; eGFR, estimated glomerular filtration rate. a The prevalence of hyperuricemia among adolescents stratified by sex. Two standards of hyperuricemia were applied (sUA $\geq 7.0 \mathrm{mg} / \mathrm{dL}$ and $\mathrm{sUA} \geq 5.5 \mathrm{mg} / \mathrm{dL}$ ). ORs of risk factors associated with hyperuricemia (sUA $\geq 7.0 \mathrm{mg} / \mathrm{dL}$ ) in univariate $(\mathbf{b})$ and multivariate (c) analysis. BMI, WC, TG, FBG, SBP and eGFR are

continuity variables. Gender (female/male), urbanization (urban/rural) and family history of gout (yes/no) are dichotomous variables. Multivariable logistic model was used to adjust for sex, BMI, WC, SBP, TG, FBG, eGFR, family history of gout and rural resident. Color bars show the significant differences $(p<0.05)$. d Manhattan plot of $-\log 10(p)$ for 35 food items associated with hyperuricemia. Bars in yellow represent the significant difference between hyperuricemia group and normal sUA group $(p<0.05)$. Bars in blue represent non-significance $(p>0.05)$

study, the overall prevalence of hypertension is high up to $21.7 \%$. However, when we defined hypertension as $\geq$ $140 \mathrm{mmHg}$ systolic or $\geq 90 \mathrm{mmHg}$ diastolic using traditional recommendation, a less strict standard, the overall prevalence

of hypertension is $7.3 \%$ in total ( $11.4 \%$ in hyperuricemia population and $5.9 \%$ in normal sUA ones).

High serum uric acid was positively associated with obesity in the overweight and obesity group in a cross-sectional 
study, which enrolled 3529 Chinese students aged 16-26 years old [11]. In another nested population cohort with a sample of 494 re-contacted adolescents from the original study, the prevalence of uric acid at risk was $37.25 \%$ and the proportion of high uric acid was $18.42 \%$, significantly higher in men than in women. Adolescents with high levels of uric acid were more likely to have abdominal obesity (OR 3.03, 95\% CI 1.38-6.64), hypertriglyceridemia (OR 4.94, 95\% CI, 2.98-8.19), and altered fasting glycemia (OR 5.15, 95\% CI, 3.42-11.05) [12]. Our data showed similar results that adolescents with enhanced BMI, increased TG, and increased FBG had higher probability of hyperuricemia occurrence in univariate and multivariate analysis.

The kidney is one of the main targets of hyperuricemia. In this study, we used the FAS-Age formula which is age based to indicate eGFR accordingly in adolescents. The current Kidney Disease Improving Global Outcomes (KDIGO) guidelines recommend the use of the bedside creatininebased Chronic Kidney Disease in Children (CKiD) equation to estimate GFR in children and the Chronic Kidney Disease Epidemiology Collaboration (CKD-EPI) equation in adults. However, this approach causes implausible changes in eGFR at the transition from pediatric to adult care [8]. Compared with CKiD and CKD-EPI formula, FAS-Age was more accurate and more suitable for our adolescent participants. In our results, the decreased eGFR was also related to high hyperuricemia incidence in the logistic regression analysis.

Diet, especially purine-rich food, are risk factors for hyperuricemia. Recently, dietary fructose intake has been increasing. It is increasing primarily from added sugars, including sucrose and high-fructose corn syrup (HFCS), and correlates epidemiologically with the rising prevalence of hyperuricemia. A total of 814 adolescents aged 13-15 years participating in the Western Australian Pregnancy Cohort (Raine) Study showed fructose intake was independently associated with serum uric acid $(p<0.01)$ in boys [13]. The results from a pooled analysis indicated that total fructose consumption was positively associated with uric acid levels in adolescents [14]. Some cross-sectional analyses and clinical trials also supported the association between HFCS-sweetened beverage intake and increased levels of circulating uric acid [15-17]. Considering the mechanism, a study indicated that fructose-rich sugar-sweetened beverage (SSB) consumption is associated with an increase in pediatric insulin resistance through the pathways in relation to visceral fat accumulation and uric acid synthesis [18]. We showed there was a highly significant difference between carbonate and SSB in consumption frequency between adolescents with hyperuricemia and with normal sUA. This result was consistent with previously reported data. Further, controlling fructose-rich beverage intake would be an effective strategy in hyperuricemia prevention.
In addition, estrogen has been reported to inhibit hypoxiainduced xanthine oxidase activity and help the kidneys get rid of uric acid [19]. The significantly higher ratio of irregular menstruation in hyperuricemia girls than in normal sUA ones indicated lower levels of estrogen in hyperuricemia girls which need to be confirmed by further clinical test.

Several limitations of the study should be noted. First, the data are cross-sectional, and therefore it is not possible to establish that relatively higher uric acid levels preceded or caused increased BMI, increased waist circumstance, increased TG, increased FBG, increased SBP and decreased eGFR. Second, the representativity of the sample population is limited, though Shandong Province is one of the most populous provinces in China. Epidemic surveys of expanded population from other south provinces need to be conducted to delegate the Chinese hyperuricemia prevalence. Third, the limitation of estimating the GFR in adolescents and young adults. More accurate eGFR estimating equations should be induced to evaluate the renal functions.

This study expands the evidence that an increasing prevalence of hyperuricemia is shown in a cohort of 9371 Chinese adolescents in a cross-sectional study. Epidemic of youth hyperuricemia may pose a future threat of gout attacks and other hyperuricemia-related diseases, which alarms the public, health professionals and health policy makers to prepare for the future health challenges.

Funding information This work financially supported by the research project grants from the National Key Research and Development Program (No. 2016YFC0903401), the National Science Foundation of China (Nos. 31900413, 81520108007, 81770869), the Shandong Province Key Research and Development Program (No. 2018CXGC1207), and the Shandong Province Natural Science Foundation (No. ZR2018ZC1053).

\section{Compliance with ethical standards}

Conflict of interest The authors declare that they have no conflicts of interest.

Open Access This article is distributed under the terms of the Creative Commons Attribution 4.0 International License (http:// creativecommons.org/licenses/by/4.0/), which permits unrestricted use, distribution, and reproduction in any medium, provided you give appropriate credit to the original author(s) and the source, provide a link to the Creative Commons license, and indicate if changes were made.

\section{References}

1. Dincer HE, Dincer AP, Levinson DJ (2002) Asymptomatic hyperuricemia: to treat or not to treat. Cleve Clin J Med 69:594, 597, 600592 passim

2. Liu R, Han C, Wu D, Xia X, Gu J, Guan H, Shan Z, Teng W (2015) Prevalence of Hyperuricemia and Gout in Mainland China from 
2000 to 2014: A Systematic Review and Meta-Analysis. Biomed Res Int 2015:762820

3. Ford ES, Li C, Cook S, Choi HK (2007) Serum concentrations of uric acid and the metabolic syndrome among US children and adolescents. Circulation 115:2526-2532

4. Zimmet P, Alberti G, Kaufman F, Tajima N, Silink M, Arslanian S, Wong G, Bennett P, Shaw J, Caprio S, International Diabetes Federation Task Force on E, Prevention of D (2007) The metabolic syndrome in children and adolescents. Lancet 369:2059-2061

5. Flynn JT, Kaelber DC, Baker-Smith CM, Blowey D, Carroll AE, Daniels SR, de Ferranti SD, Dionne JM, Falkner B, Flinn SK, Gidding SS, Goodwin C, Leu MG, Powers ME, Rea C, Samuels J, Simasek M, Thaker VV, Urbina EM Subcommittee On S, Management of high blood pressure In C (2017) Clinical practice guideline for screening and management of high blood pressure in children and adolescents. Pediatrics 140(3), e20171904

6. Whelton PK, Carey RM, Aronow WS, Casey DE Jr, Collins KJ, Dennison Himmelfarb C, DePalma SM, Gidding S, Jamerson KA, Jones DW, MacLaughlin EJ, Muntner P, Ovbiagele B, Smith SC Jr, Spencer CC, Stafford RS, Taler SJ, Thomas RJ, Williams KA Sr, Williamson JD, Wright JT Jr (2018) 2017 ACC/AHA/AAPA/ABC/ ACPM/AGS/APhA/ASH/ASPC/NMA/PCNA Guideline for the prevention, detection, evaluation, and management of high blood pressure in adults: a report of the American College of Cardiology/ American Heart Association Task Force on Clinical Practice Guidelines. J Am Coll Cardiol 71:e127-e248

7. Pottel H, Hoste L, Dubourg L, Ebert N, Schaeffner E, Eriksen BO, Melsom T, Lamb EJ, Rule AD, Turner ST, Glassock RJ, De Souza V, Selistre L, Mariat C, Martens F, Delanaye P (2016) An estimated glomerular filtration rate equation for the full age spectrum. Nephrology, dialysis, transplantation: official publication of the European Dialysis and Transplant Association - European Renal Association 31:798-806

8. Pottel H, Bjork J, Bokenkamp A, Berg U, Asling-Monemi K, Selistre L, Dubourg L, Hansson M, Littmann K, Jones I, Sjostrom P, Nyman U, Delanaye P (2019) Estimating glomerular filtration rate at the transition from pediatric to adult care. Kidney Int 95: 1234-1243

9. Baldree LA, Stapleton FB (1990) Uric acid metabolism in children. Pediatr Clin N Am 37:391-418

10. Loeffler LF, Navas-Acien A, Brady TM, Miller ER 3rd, Fadrowski JJ (2012) Uric acid level and elevated blood pressure in US adolescents: National Health and Nutrition Examination Survey, 19992006. Hypertension 59:811-817
11. Duan Y, Liang W, Zhu L, Zhang T, Wang L, Nie Z, Chen Y, He L, Jin Y, Yao Y (2015) Association between serum uric acid levels and obesity among university students (China). Nutr Hosp 31:24072411

12. Serrano N, Ojeda CA, Gamboa Delgado E, Colmenares Mejia CC, Quintero Lesmes DC (2019) Uric acid and its association with the components of the metabolic syndrome in Colombian adolescents. Nutr Hosp 36:325-333

13. Bobridge KS, Haines GL, Mori TA, Beilin LJ, Oddy WH, Sherriff J, O'Sullivan TA (2013) Dietary fructose in relation to blood pressure and serum uric acid in adolescent boys and girls. J Hum Hypertens 27:217-224

14. Jamnik J, Rehman S, Blanco Mejia S, de Souza RJ, Khan TA, Leiter LA, Wolever TM, Kendall CW, Jenkins DJ, Sievenpiper JL (2016) Fructose intake and risk of gout and hyperuricemia: a systematic review and meta-analysis of prospective cohort studies. BMJ Open 6:e013191

15. Choi JW, Ford ES, Gao X, Choi HK (2008) Sugar-sweetened soft drinks, diet soft drinks, and serum uric acid level: the Third National Health and Nutrition Examination Survey. Arthritis Rheum 59: 109-116

16. Bomback AS, Derebail VK, Shoham DA, Anderson CA, Steffen LM, Rosamond WD, Kshirsagar AV (2010) Sugar-sweetened soda consumption, hyperuricemia, and kidney disease. Kidney Int 77: 609-616

17. Stanhope KL, Medici V, Bremer AA, Lee V, Lam HD, Nunez MV, Chen GX, Keim NL, Havel PJ (2015) A dose-response study of consuming high-fructose corn syrup-sweetened beverages on lipid/ lipoprotein risk factors for cardiovascular disease in young adults. Am J Clin Nutr 101:1144-1154

18. Lin WT, Chan TF, Huang HL, Lee CY, Tsai S, Wu PW, Yang YC, Wang TN, Lee CH (2016) Fructose-Rich Beverage Intake and Central Adiposity, Uric Acid, and Pediatric Insulin Resistance. J Pediatr 171:90-96 e91

19. Budhiraja R, Kayyali US, Karamsetty M, Fogel M, Hill NS, Chalkley R, Finlay GA, Hassoun PM (2003) Estrogen modulates xanthine dehydrogenase/xanthine oxidase activity by a receptorindependent mechanism. Antioxid Redox Signal 5:705-711

Publisher's note Springer Nature remains neutral with regard to jurisdictional claims in published maps and institutional affiliations.? 